\title{
Qualitätsmanagement: Ein Werkzeug für die Wettbewerbsfähigkeit
}

\author{
Bernardo de Sousa*
}

Vor 10 Jahren schrieb der berühmte Management-BeraterTom Peters, zusammen mit Bob Waterman, einen Bestseller mit dem Titel 'Auf der Suche nach Spitzenleistungen'. In diesem Buch analysierten die Autoren 43 Spitzenfirmen der USA und beschrieben die Faktoren, welche diese Firmen zum Erfolg führten: es handelt sich um acht Erfolgsfaktoren. Drei Jahre später publizierte er, zusammen mit Nancy Austin, ein neues Buch, mit dem Titel 'Leistung aus Leidenschaft', in welchem er die Anzahl der Erfolgsfaktoren auf drei reduzierte. 1988 gab er ein neues Buch 'Kreatives Chaos' heraus. In diesem Buch sprach er nun von einem einzigen Erfolgsfaktor: Qualität. Es freut mich sehr, über dieses Thema referieren zu dürfen, vor allem weil ich überzeugt bin, dass es uns nur durch die Implementierung von Qualitätsmanagement-Konzepten gelingen wird, unsere Wettbewerbsfähigkeit und unseren unternehmerischen Erfolg zu sichern. deln:

Ich schlage vor, drei Punkte zu behan-

*Korrespondenz: Dr. B. de Sousa

Corporate Quality Management

Ciba-Geigy $A G$

$\mathrm{CH}-4002$ Basel
1) Geschichtliches

2) TQM heute

3) Die Ciba-Geigy-Strategie zur Implementierung von TQM

Zuerst werden wir einen Blick auf die geschichtliche Entwicklung werfen.

Dann möchte ich den Status von TQM und wie Qualität und TQM heute überhaupt verstanden werden mit Ihnen besprechen.

Abschliessend erläutere ich Ihnen die Ciba-Geigy-Strategie zur Implementierung von TQM im ganzen Konzern.

Auf Grund der begrenzten Zeit, die mir zur Verfügung steht, können diese drei Aspekte nur kurz besprochen werden.

\section{Geschichtliches}

\section{a) Der Einmann-Betrieb}

Vor der industriellen Revolution lag die Qualität in den Händen des Handwerkers: Der Handwerker erlernte seinen Beruf bei einem Meister und nach erfolgter Lehre machte er sich selbständig oder, im Falle eines grösseren Betriebes, blieb er bei seinem Meister. Ein Kunde bestellte einen Stuhl oder einen Tisch direkt beim Schreiner; sämtliche Abläufe wie etwa Design, Konstruktion oder Produktion, Logistik und Marketing sowie die Qualität des Produktes und der Dienstleistun-

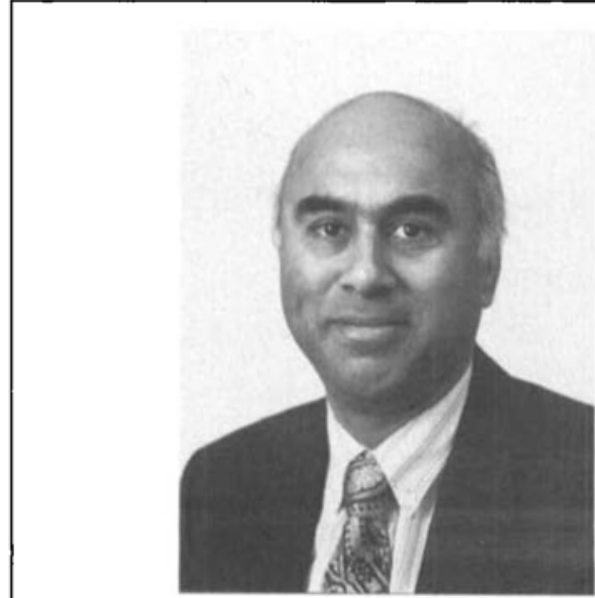

Bernardo de Sousa: Geb. 13. August 1945 in Aldona, Goa, Indien, Bürger von Riehen (BS). Ausbildung: 1960: Secondary School Certificate (Poona Board) der Loyola High School, Margaum, Goa. 1964: B.Sc. (Hons.) (Bombay University) im St. Xavier's College, Bombay. 1974: Dr. rer. nat. (Universität Fribourg, Schweiz). Doktorarbeit in der org. Photochemie bei Prof. Dr. E. Giovannini.

Aufgaben: 1970-1975: Assistent im Institut für Organische Chemie, Universität Fribourg, Schweiz. 1975-1976: Post-Doktor-Arbeit über Synthesen und Reaktionsmechanismen auf dem Gebiet der optischen Aufheller bei Prof. Dr. A.E. Siegrist. 1977-1982: CibaGeigy, Division Farbstoffe und Chemikalien, Departement Forschung und Entwicklung. Syntheseforschung auf den Gebieten Mottenschutzmittel, antimikrobielle Mittel, neutrale Leime für Papier, Anthelmintika. 1982-1985: Leiter des Bereiches 'Gesetzgebung', in der Abteilung Produktesicherheit. 1985-1989: Leiter der Abteilung Produktesicherheit, Division Farbstoffe und Chemikalien. Dez 1989: Beauftragter der Konzernleitung für Qualität (Corporate Quality Officer)

gen hatte dieser Schreiner oder ganz allgemein der Handwerker im Griff.

\section{b) Die industrielle Revolution}

Mit der industriellen Revolution entstanden die Fabriken, welche auch weniger geschultes Personal anstellten. Die Handwerker und deren Meister wurden teils Fabrikarbeiter, teils Aufseher - im heutigen Sprachgebrauch würden sie Spezialisten, Kader oder Consultants genannt. 


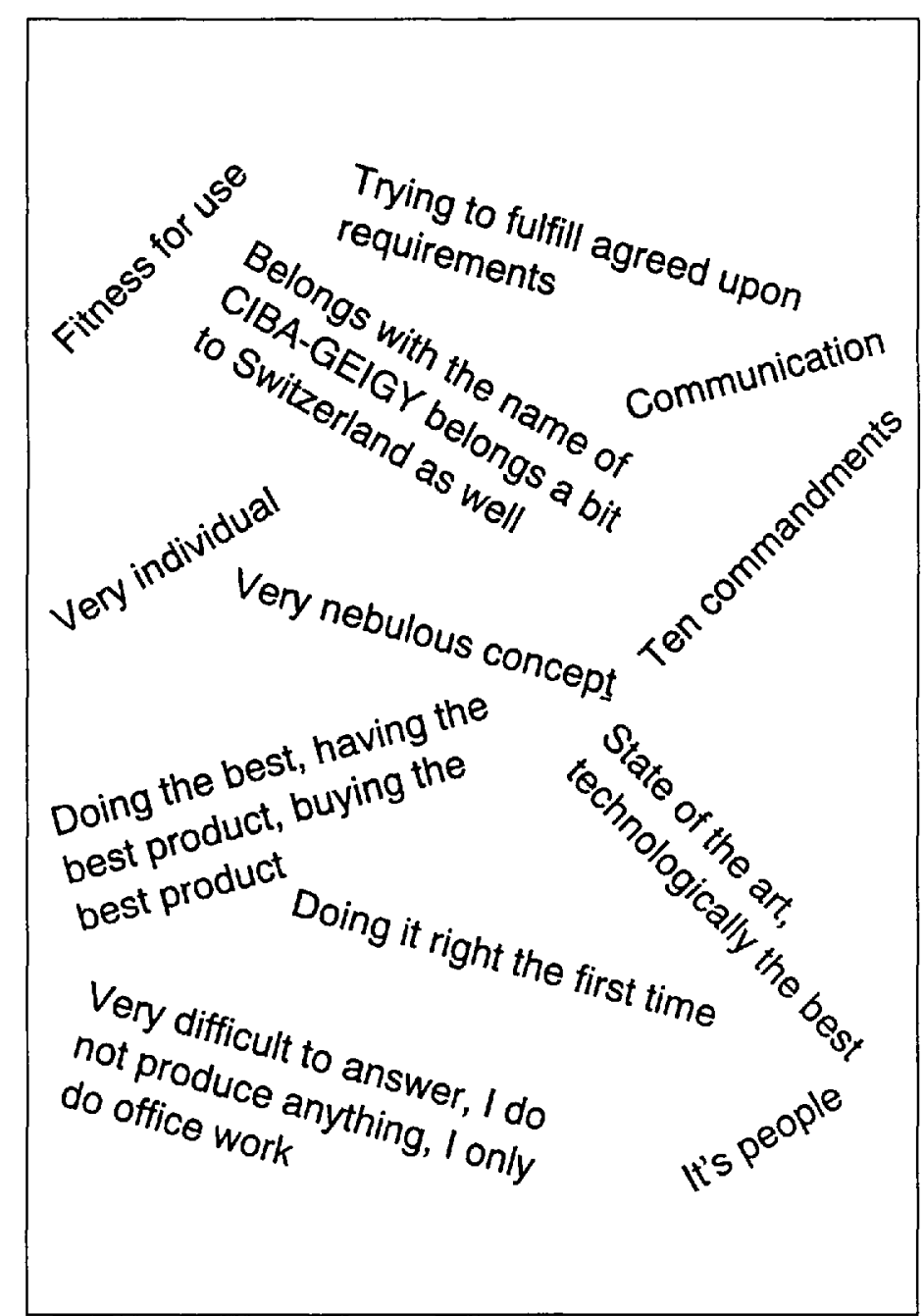

Fig. 1. What is your perception of quality within Ciba-Geigy?

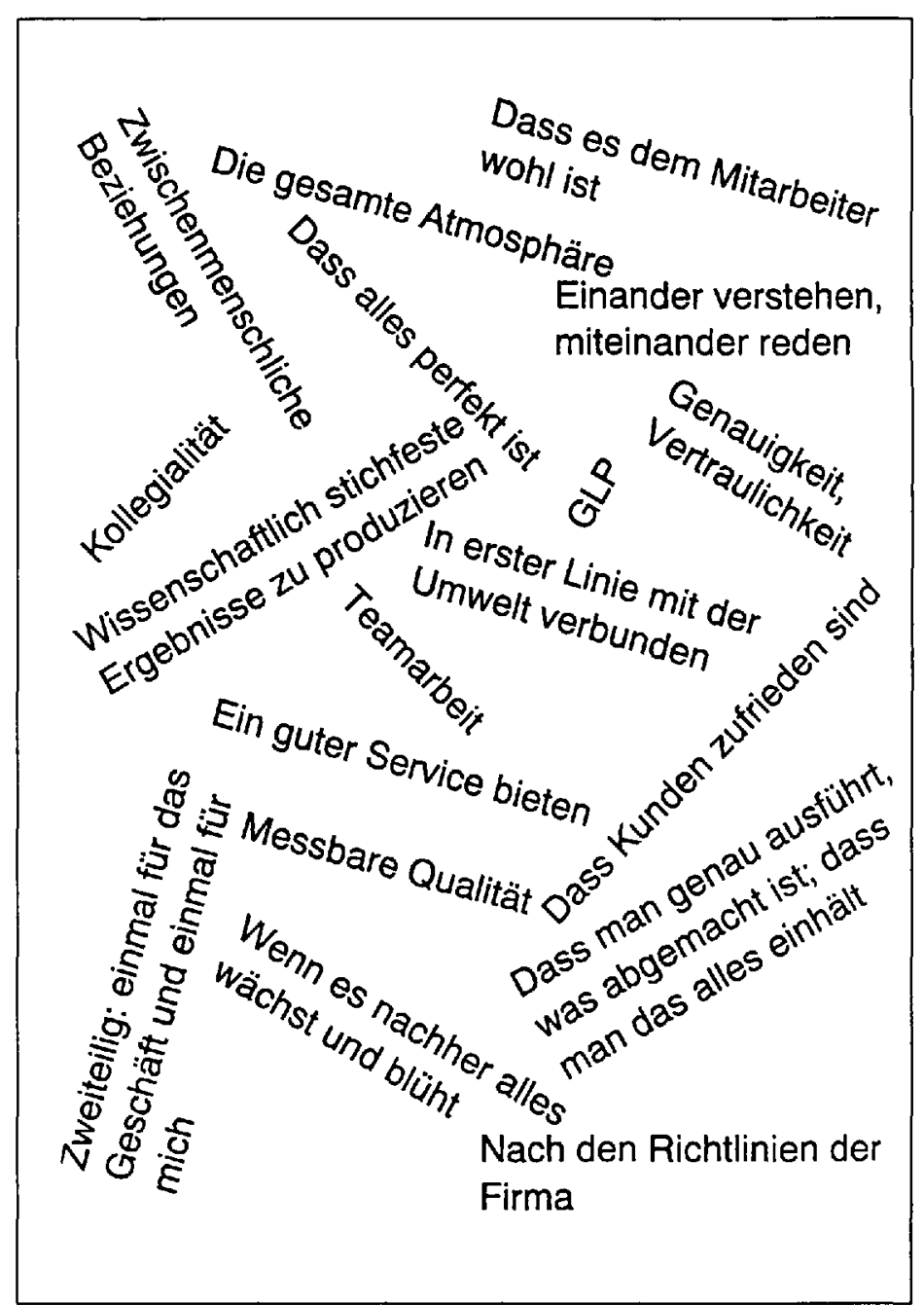

Fig. 2. Was bedeutet für Sie Qualität am Arbeitsplatz?
Fig. 3. Was bedeutet für SieQualität amArbeitsplatz?
Qualität wurde eine Inspektionsaufgabe der Fertigware durch diese Spezialisten.

c) Das Taylorsche System

Im späteren 19. Jahrhundert wurde das von Fredrick E. Taylor propagierte System - das sogenannte Taylorsche-System - eingeführt. Dieses System hatte zum Ziel, die Produktivität durch die Einführung einer rigiden Hierarchie zu erhöhen. Die Rolle der Kontrollen, sowohl der Mitarbeiter als auch der hergestellten Produkte, wurde somit institutionalisiert und gestärkt.

\section{d) Statistische Qualitätskontrolle}

In den 20er Jahren wurden in den Bell Laboratories in USA einige statistische Werkzeuge propagiert, wie etwa die von Walter Shewhart entwickelten Kontrollkarten oder die Anwendung der Wahrscheinlichkeitstheorie bei der Inspektion von Produktmustern. Die berühmten Qualitätsexperten Deming und Juran waren damals beide junge Ingenieure und stammen aus dieser Schule.

\section{e) Der 2. Weltkrieg}

Der 2. Weltkrieg brachte eine Ausdehnung der bereits während des ersten Welt- 


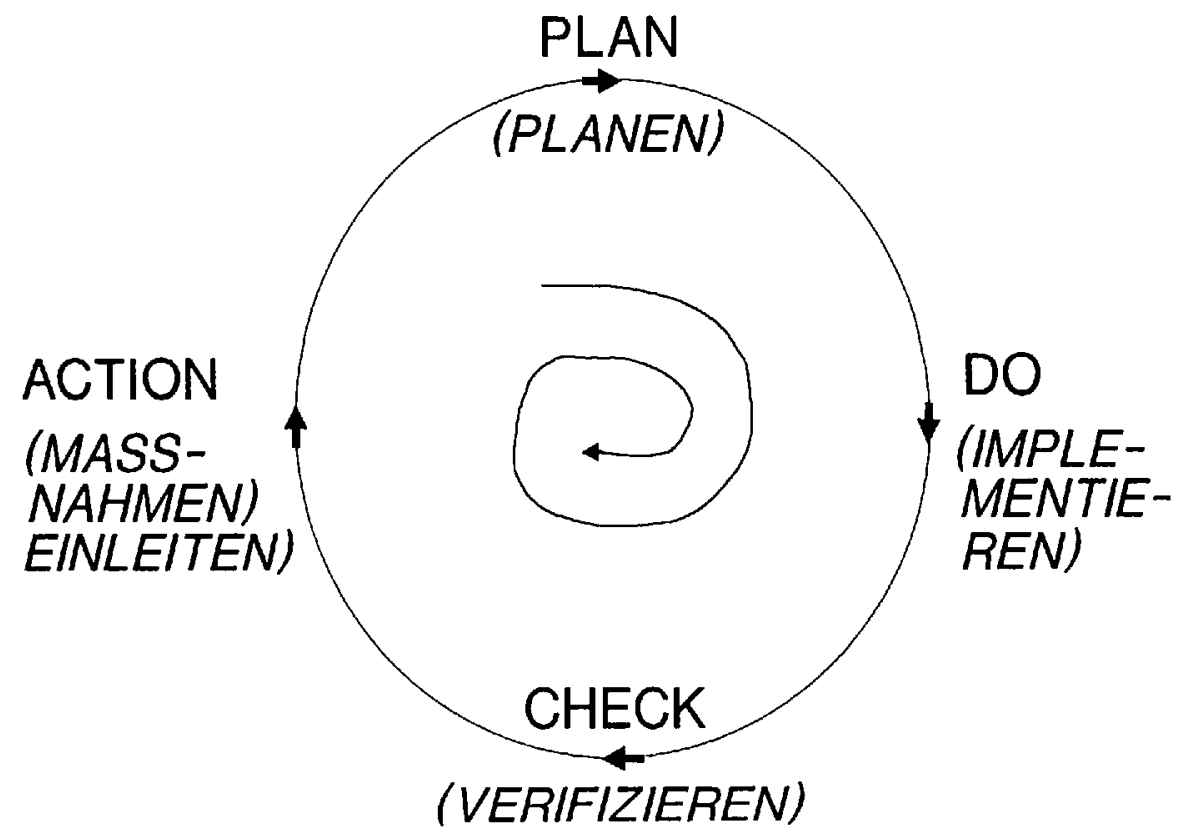

Fig. 4. Der Deming-Kreis (PDCA)

krieges eingeführten Inspektion (Qualitätskontrolle) mit sich, vor allem für Militärartikel, und wurde durch die Einführung des Militärstandards MIL-STD-105 konkretisiert. Es wurden auch um diese Zeit in USA zum ersten Mal 8tägige Statistikkurse für junge Ingenieure etabliert. Ebenfalls um diese Zeit wurde eine neue organisatorische Einheit im TaylorschenSystem eingegliedert, die Abteilung für Qualitätskontrolle. Auch Vereinigungen zur Förderung der Qualität stammen aus dieserZeit: die American Society for Quality Control z.B. wurde 1946 gegründet.

\section{f) Nach dem 2. Weltkrieg}

Der Bedarf an sämtlichen Gütern war gross nach dem 2. Weltkrieg. Dementsprechend war die erste Priorität dem Produktionsvolumen gewidmet, die Qualität verlor an Bedeutung: Wenn die Nachfrage viel grösser ist als das Angebot, so sinkt in der Regel die Qualität der Produkte und der Dienstleistungen. Aber die Rolle der Abteilung Qualitätskontrolle und der Inspektion wuchs, da die Unternehmen, trotz einer Betonung auf das Produktionsvolumen, doch ein gewisses Minimum an Qualität benötigten.

\section{g) Qualitätssicherung}

Mit der massiven Erhöhung der Nachfrage und der Produktivität wurde es immer schwieriger, Qualität nur mit Inspektionen in den Griff zu bekommen, nicht zuletzt, weil dies einen massiven Zuwachs an Inspektionspersonal bedeutete. Um dieses Problem zu überwinden, wurde ein neues Konzept eingeführt, das der Quali- tätssicherung. In USA konkretisierte sich dieses Konzept als die berühmte Militärnorm MIL-Q-9858, die von vielen Ländern sowie von der NATO übernommen wurde und die Basis der heutigen ISONorm 9000er Reihe sein dürfte.

\section{h) Die japanische Revolution}

Die japanische Industrie, die vor allem die Militärmacht belieferte, musste nach dem 2. Weltkrieg umfunktioniert werden - eine Situation die heute im ehemaligen Ostblock zu finden ist. Sie war aber für diese Aufgabe schlecht vorbereitet und 'Made in Japan' wurde synonym mit ganz schlechter Qualität. Es war in jener Zeit, Ende der 40er Jahre, dass der amerikanische Statistiker W. Edwards Deming, im Zusammenhang mit dem Marshall Plan in Japan als Berater ankam. Seine Konzepte, die in den USA kaum Anhänger fanden, wurden in Japan mit Enthusiasmus aufgenommen - niemand ist ja Prophet im eigenen Lande. Das Zitat von Deming 'Take care of quality and productivity will take care of itself' (Verbessere die Qualität und die Produktivität wird sich von sich aus verbessern) ist vielen von Ihnen sicherlich bekannt. Ebenfalls bekannt ist die Tatsache, dass durch die Umsetzung der Konzepte von Deming und Juran, der auch kurz nach Deming in Japan beratend wirkte, die japanische Industrie einen sehr hohen Standard erreichte.

\section{i) Qualitätsmanagement als weltweiter Begriff}

Die japanische Wirschaftsrevolution hatte grosse Konsequenzen in USA: mas- sive Marktanteilverluste z.B. in der Automobilindustrie und Elektronikbranche. Eine Folge davon ist nämlich, dass sich seit Anfang der 80er Jahre, die Konzepte von Deming, Juran sowie anderer Qualitätsexperten grosser Beliebtheit erfreuen. Heute haben verschiedene Unternehmen wie IBM oder Disneyworld TQM eingeführt und vielen Firmen wie Xerox oder Milliken, ist es gelungen auf diesem Wege verlorene Marktanteile wieder zu gewinnen.

In Europa war anfänglich Qualitätsmanagement nur punktuell hier und dort zu finden. Ein europaweiter Vorstoss wurde 1988, nach einer Initiative von Jacques Delors, Präsident der Europäischen Gemeinschaft, mit der Gründung der European Foundation for Quality Management (E.F.Q.M.) gemacht. Wir dürfen stolz sein, dass 3 der 14 Gründungsmitglieder von E.F.Q.M. Schweizer Unternehmen sind. Heute zählt diese Organisation bereits mehr als 220 Mitglieder in ganz Europa.

\section{Total Quality Management heute}

Wir sehen also, dass Qualitätsmanagement und Qualität im allgemeinen heute weltweit propagiert und implementiert wird. Was verstehen wir aber unter dem Begriff 'Qualität'?

\section{a) Definition der Qualität}

In unserer Firma führten wir vor zwei Jahren auf internationaler Ebene spontane Interviews durch, wo wir die direkte Frage stellten: 'Was verstehen Sie unter Quali- 
tät'? In einer analogen Übung in Basel, letztes Jahr, fragten wir die Mitarbeiter, wiederum spontan, was sie unter Qualität am Arbeitsplatz verstehen. Einige Antworten sehen sie in den Fig. 1-3.

In der täglichen Presse und vor allem in der Werbung findet man viele andere Definitionen und Werbeslogans wie etwa 'Qualität ist unsere Stärke', 'Da weiss man, was man hat' oder ähnliches. Eine Definition, die aus einer deutschen Bierbrauerei stammt und mir ganz besonders gefällt ist: 'Qualität ist, wenn der Kunde zurückkommt und nicht das Produkt'.
Wir können uns vorstellen, dass Kunden dann zurückkommen, wenn sie zufrieden sind und sie werden zufrieden sein, wenn die Anforderungen die sie stellen lückenlos erfüllt werden.

'Qualität heisst, Erfüllung der vereinbarten Anforderungen bei minimalen Kosten'.

Somit sind sowohl eine Plastikuhr als auch eine teure Markenuhr, ein Kleinauto als auch ein Rolls Royce Qualitätsartikel, wenn diese die Kundenanforderungen erfüllen. Diese, vor allem von Philip Crosby propagierte Definition, findet heute am

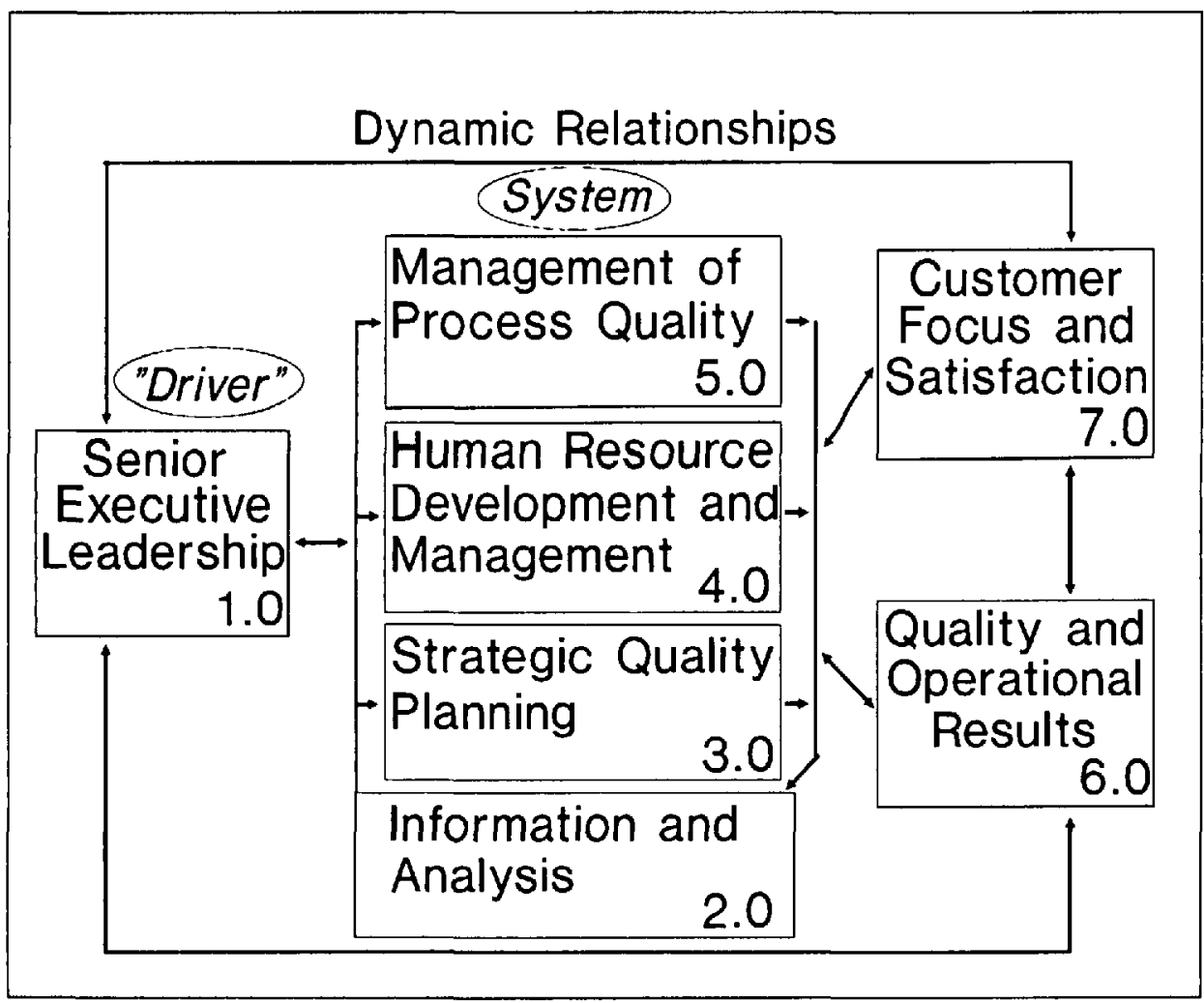

Fig. 5. Baldrige Award Criteria Framework

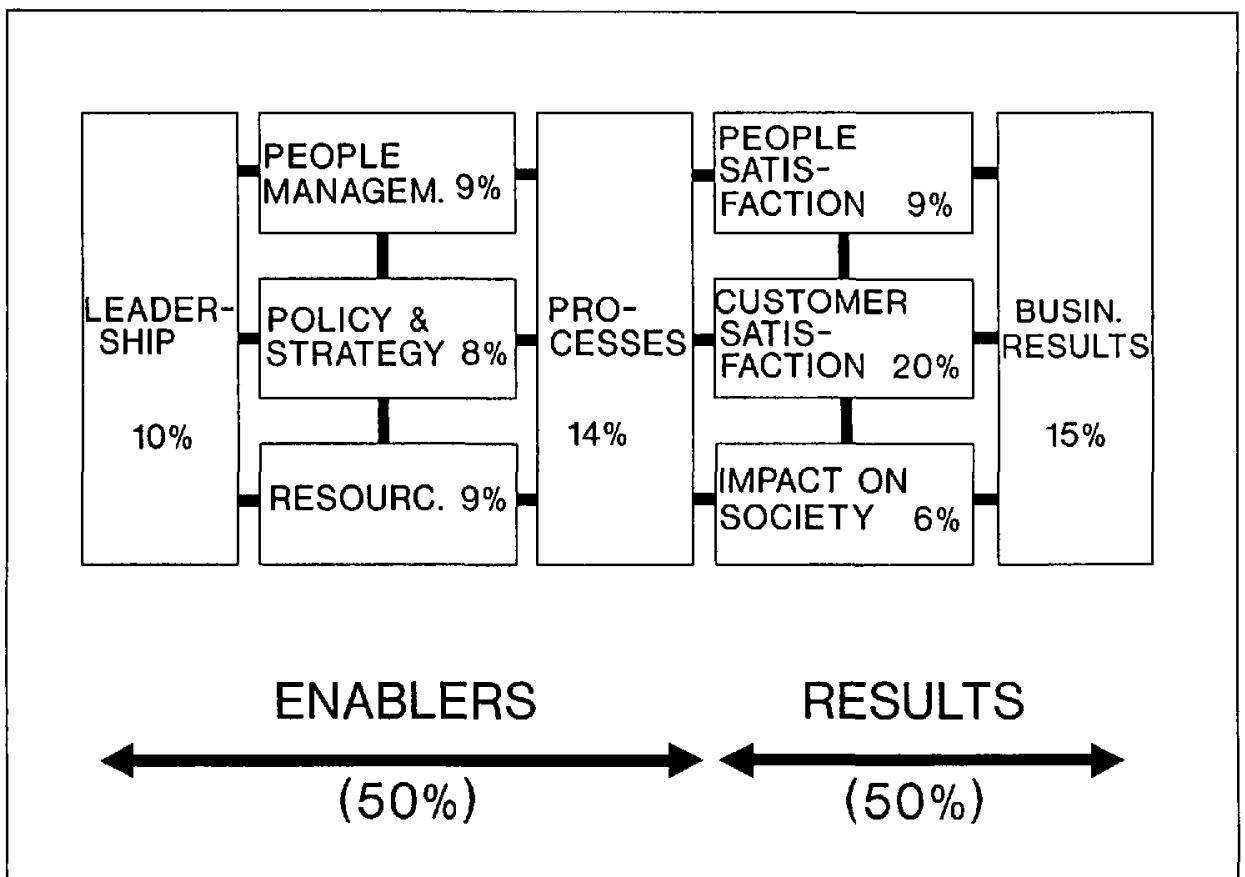

Fig. 6. The European TQM Model meisten Verwendung. Selbstverständlich haben verschiedene Experten verschiedene Ansichten, die ich Ihnen hier kurz vorstellen möchte.

William Edwards Deming, wie bereits gesagt, ein Schüler des Statistikers Walter $A$. Shewhart, verzichtet auf eine übergreifende Definition von Qualität, da ein Produkt in der Regel zahlreiche Merkmale zahlreiche Qualitäten - aufweist. Um sich längerfristig zu bewähren, muss ein Produkt kontinuierlich verbessert werden und zwar gemäss dem sogenannten DemingKreis: Zuerst muss jede Aktivität geplant werden, anschliessend wird sie implementiert. In einem nächsten Schritt wird verifiziert, ob der Ist-Zustand mit dem Soll übereinstimmt und die Abweichungen identifiziert. Anschliessend werden die nötigen Korrekturmassnahmen eingeleitet, um die Abweichungen zu beseitigen. Jetzt wird wieder neu geplant, allerdings mitengeren Spezifikationen, und der Kreis wird auf diese Art kontinuierlich weitergeführt (Fig. 4).

Deming hat sein Konzept in 14 Punkten niedergelegt, in welchen er auf die Statistik grossen Wert legt. Er betont auch die Notwendigkeit, Kommunikation sowie ein offenes Klima zu fördern. Die Konzepte von Deming sind eindeutig Produkt orientiert, die 'soft'-Faktoren wie etwa Motivation, Führungsart, Anerkennung werden kaum berücksichtigt.

Joseph M. Juran definiert Qualität als 'fitness for use', was zwei Aspekte beinhaltet:

1) Produkteleistungen, die zur Kundenzufriedenheit führen und

2) Abwesenheit von Produktmängeln, wodurch eine Kundenunzufriedenheit vermieden wird.

Bei der Entwicklung eines Produktes sind verschiedene Personen und Abteilungen involviert, alle mit unterschiedlichen Anforderungen an dieses Produkt. Sie sind alle Kunden, dementsprechend müssen interne Kunden, d.h. die involvierten Mitarbeiter des Unternehmens und externe Kunden, d.h. die Endabnehmer, berücksichtigt werden. Juran führte den Begriff 'Qualitätskosten' ein und betonte die Notwendigkeit einer kontinuierlichen Verbesserung durch die drei Schritte: Planung - Kontrolle - Verbesserung, was er als die Juran-Trilogie bezeichnet.

Armand Feigenbaum legt viel Wert auf die horizontale Kommunikation bei allen Aktivitäten eines Unternehmens. Er meinte, dass 'the first principle to recogni$\mathrm{ze}$ is that quality is everyboby's job'. Für Feigenbaum wird Qualität durch die Erwartungen des Verbrauchers bestimmt.

Philip B. Crosby definiert Qualität als die Erfüllung von Anforderungen, die zu- 
erst definiert werden müssen. Dies gilt für alle involvierten Personen innerhalb des Unternehmens, als auch extern. Qualität wird durch Vorbeugung und nicht durch Inspektionen und Kontrollen erreicht. Als Leistungsstandard gilt das Null-FehlerPrinzip. Schliesslich meint Crosby, dass Qualität gemessen werden kann und muss, wobei sich die Kosten der Qualität aus der Summe der Kosten der Erfüllung der Anforderungen, der Vorbeugung und der Nicht-Erfüllung der Anforderungen ergeben. Für Crosby ist Qualität eine Sache der Einstellung. Die vier Prinzipien von Crosby wie auch seine Bücher sind einfach zu verstehen, wodurch seine Konzepte sehr populär wurden. Seine grosse Schwäche liegt darin, dass er keine Werkzeuge anbietet, wie diese Konzepte eingeführt werden sollen. In seinen Büchern ist z.B. von der Statistik kaum die Rede.

Ein letzter Experte den ich hier erwähnen möchte, ist der Japaner Kaoru Ishika$w a$, der u.a. wegen seines sogenannten Ishikawa oder Ursache-Wirkung-Diagramms sehr bekannt wurde. Er definiert zwar Qualität nicht explizite, beschreibt sie aber als eine Funktion von sechs Faktoren:

1) Die Qualitätkommt zuerst (quality first)

2) Erfüllung der Anforderungen der Konsumenten (conformance to consumers' requirements)

3) Interfunktionelle Mitwirkung

4) Kontinuierliche Verbesserung

5) Partizipatives Management

6) Die soziale Verantwortung eines Unternehmens

Wie Sie sehen können, weisen diese fünf Experten unterschiedliche Nuancen in ihren Auffassungen über Qualität und Qualitätsmanagement auf. Sowohl Deming als auch Juran sind produktorientiert; Juran und Crosby sprechen von internen und externen Kunden sowie von Qualitätskosten, Feigenbaum und Ishikawa betonen die Notwendigkeit der interfunktionellen oder horizontalen Kommunikation.

Alle fünf Qualitätspäpste sind sich aber über eines einig: alle betonen die Wichtigkeit der Verpflichtung und Unterstützung durch das Top-Management. Die bis heute gewonnene Erfahrung gibt ihnen recht: Überall dort, wo das Top-Management versucht hat, nur mit Lippenbekenntnis Qualitätsmanagement einzuführen, endete es mit einem Misserfolg.

Es gibt noch andere Experten und Consultants mit weiteren Ansichten, aber es dürfte Ihnen klar sein, dass ein Unternehmen mit der Absicht TQM einzuführen zunächst mit der folgenden Frage konfrontiert wurde: 'Mit welchem Konzept von welchem Experten fange ich nun an?'

Tabelle. TQM Results: Some Examples

\begin{tabular}{|c|c|c|}
\hline & Before & After \\
\hline $\begin{array}{l}\text { Market Share } \\
\text { Carpet Dyes, DC Div., Greensboro, USA }\end{array}$ & $\begin{array}{l}\text { 1988: } \\
\text { our Share } 46,6 \%\end{array}$ & $\begin{array}{l}\text { 1989: } \\
\text { our Share } 50,5 \% \\
\text { Tools: Partnership }\end{array}$ \\
\hline $\begin{array}{l}\text { OFF-Spec Material } \\
\text { Clayton Aniline Co., Manchester }\end{array}$ & $1985:>8 \%$ & $\begin{aligned} & \text { 1990: } \text { ca. } 1 \% \\
& \text { Tools: Prevention } \\
& \\
& \text { Error Cause Removal }\end{aligned}$ \\
\hline $\begin{array}{l}\text { Inventory } \\
\text { Reduction, DC Div., USA }\end{array}$ & $\begin{array}{l}\text { 1984: } \quad \rightarrow \\
\text { Reduction by } 20 \mathrm{~N} \\
\text { Cashflow Increase }\end{array}$ & $\begin{array}{l}\text { 1986: } \\
\text { M USS } \\
2 \text { MM USS } \\
\text { Tools: Def. of Requirements } \\
\text { Process Model }\end{array}$ \\
\hline Billing Errors, DC Div. Greensboro, USA & $1984: c a .8 \%$ & $\begin{aligned} \text { 1986: } & \text { ca. } 2 \% \\
\text { Tools: } & \text { Prevention } \\
& \text { Zero Defects }\end{aligned}$ \\
\hline EC Prduction Registration, DC Div., Basel & $\begin{array}{l}\text { 1986: } \\
\text { 23,8 Months } \\
4 \text { Dossiers }\end{array}$ & $\begin{array}{l}\text { 1988: } \\
13 \text { Months } \\
10 \text { Dossiers } \\
\text { Tools: Benchmarking } \\
\quad \text { Flow Chart }\end{array}$ \\
\hline $\begin{array}{l}\text { No. of Packages } v s \text {. Machine Hours } \\
\text { PH Div. Argentina }\end{array}$ & 1987: 1245 & $\begin{array}{ll}\text { 1988: } & 1874 \\
\text { Tools: } & \text { Ishikawa } \\
& \text { Pareto }\end{array}$ \\
\hline
\end{tabular}

Diese Situation führte sogar zu einer Bereicherung der Qualitätsterminologie: TQC oder Total Quality Control, wie die Japaner Qualitätsmanagement nennen, wurde in Total Quality Chaos umbenannt!

In der Praxis fingen die meisten Firmen mit einem oder zwei Experten an und entwickelten mit der Zeit eine für sie geeignete und massgeschneiderte Methode.

Eine neue Entwicklung entstand Mitte der 80er Jahre. Nachdem die amerikanische Industrie an allen Fronten massive Marktverluste eingebüsst hatte, beschloss die US Regierung dringend, konkrete Massnahmen zu treffen, um deren Wettbewerbsfähigkeit wieder zurückzugewinnen. Zweifellos war Qualität eines der wichtigsten Vehikel, dieses Ziel zu erreichen. Das National Institute of Standards and Technology (NIST) wurde beauftragt, ein Qualitätsmanagement Modell zu entwickeln. Nach Konsultation mit sämtli- chen Experten auf dem Gebiet des Qualitätsmanagements entstand auf diese Art 1988 der heute berühmte Malcolm Baldrige National Quality Award. Der Award per se ist meines Erachtens für unsere Betrachtungen nicht von grossem Interesse. Viel wichtiger ist die Tatsache, dass wir zum ersten Male ein greifbares Qualitätsmanagement-Modell in den Händen hatten. Dieses Modell verzichtet auf eine Definition von Qualität. Es nimmt aber Elemente aus allen Konzepten und beschreibt, was ein Unternehmen tun sollte, um wettbewerbsfähig zu werden (Fig. 5).

Dieses Modell hat sieben Kategorien: Führung, Informationen und Analyse, Strategische Qualitätsplanung, Personalentwicklung und -führung, Prozess-Management, Qualitäts- und operationelle Ergebnisse und Kundenorientierung/Kundenzufriedenheit. Jede Kategorie ist in sogenannte 'Examination Items' unterteilt und 
jedes 'Item' in 'Areas to Address'. Es gibt total 28 'Examination Items' und 89 'Areas to Address'.

Die Führung hat die Aufgabe, sich die nötigen Informationen zu beschaffen und zu analysieren, womit eine korrekte Strategie geplant wird. Mit motivierten Mitarbeiterinnen und Mitarbeitern werden dann die Abläufe festgehalten und durchgeführt. Sämtliche Abläufe werden auf Grund der erzielten Resultate gemessen und verbessert, was schliesslich zur Kundenorientierung und Kundenzufriedenheit führt. Bitte merken Sie sich, dass das Ziel nicht alleine die Kundenzufriedenheit ist, sondern auch Kundenzufriedenheit im Vergleich zur Konkurrenz und eine Erhöhung des Marktanteils.

Das Malcolm-Baldrige-Modell wird jedes Jahr im Sinne einer kontinuierlichen Verbesserung revidiert. Dieses Modell hat aber doch gewisse Schwächen: es kommen z.B. Verantwortung eines Unternehmens gegenüber der Umwelt oder auch die finanziellen und nicht-finanziellen unternehmerischen Ergebnisse zu kurz.

Im Januar dieses Jahres wurde das von E.F.Q.M. entwickelte TQM-Modell veröffentlicht, in welchem diese Schwächen eliminiert worden sind. Dieses Modell bildet die Basis des Europäischen QualitätsAwards, welche von der EG, EOQ, EFTA und selbstverständlich auch von der E.F.Q.M. unterstützt wird. Das europäische TQM-Modell hat neun Kategorien (Fig. 6); Sie werden bemerken, dass die Resultate in diesem Modell stark betont werden. Qualität ist keine Religion, sondern ein Vehikel für Unternehmen, wett- bewerbsfähiger und profitabler zu werden. Sollte TQM nicht zu besseren Ergebnissen führen, wozu nützt dann ja die ganze Übung?

\section{Die Ciba-Geigy-Strategie zur Implemetierung von TQM}

1982 führte die ehemalige Division Farbstoffe und Chemikalien in Greensboro, USA, als erste Ciba-Geigy-Einheit Total Quality Management ein. Andere Einheiten folgten: z.B. die Division Farbstoffe und Chemikalien in Basel, die Konzerngesellschaften in Mexiko, Italien, Argentinien und sogar an der Elfenbeinküste. Im Oktober 1989 beschloss die Konzemleitung, Qualitätsmanagement weltweit im ganzen Konzern einzuführen.

Bei der Bestimmung einer Strategie, TQM im Konzern einzuführen, mussten wir die folgenden drei Faktoren berücksichtigen:

1) Der Ciba-Geigy-Konzern ist ein heterogenes Gebilde: wir haben viele Divisionen mitsehrverschiedenen Produkten und Dienstleistungen, in sehr verschiedenen Ländern, mit unterschiedlichen Sprachen, Kulturen und Mentalitäten.

2) Einige Einheiten hatten bereits mit eigenen Programmen angefangen; diese mussten in der Strategie berücksichtigt werden.

3) Qualität ist eine Aufgabe der Linie. In keinem Falle wollten wir eine grosse zentrale Stabstelle für Qualitätsmanagement schaffen.

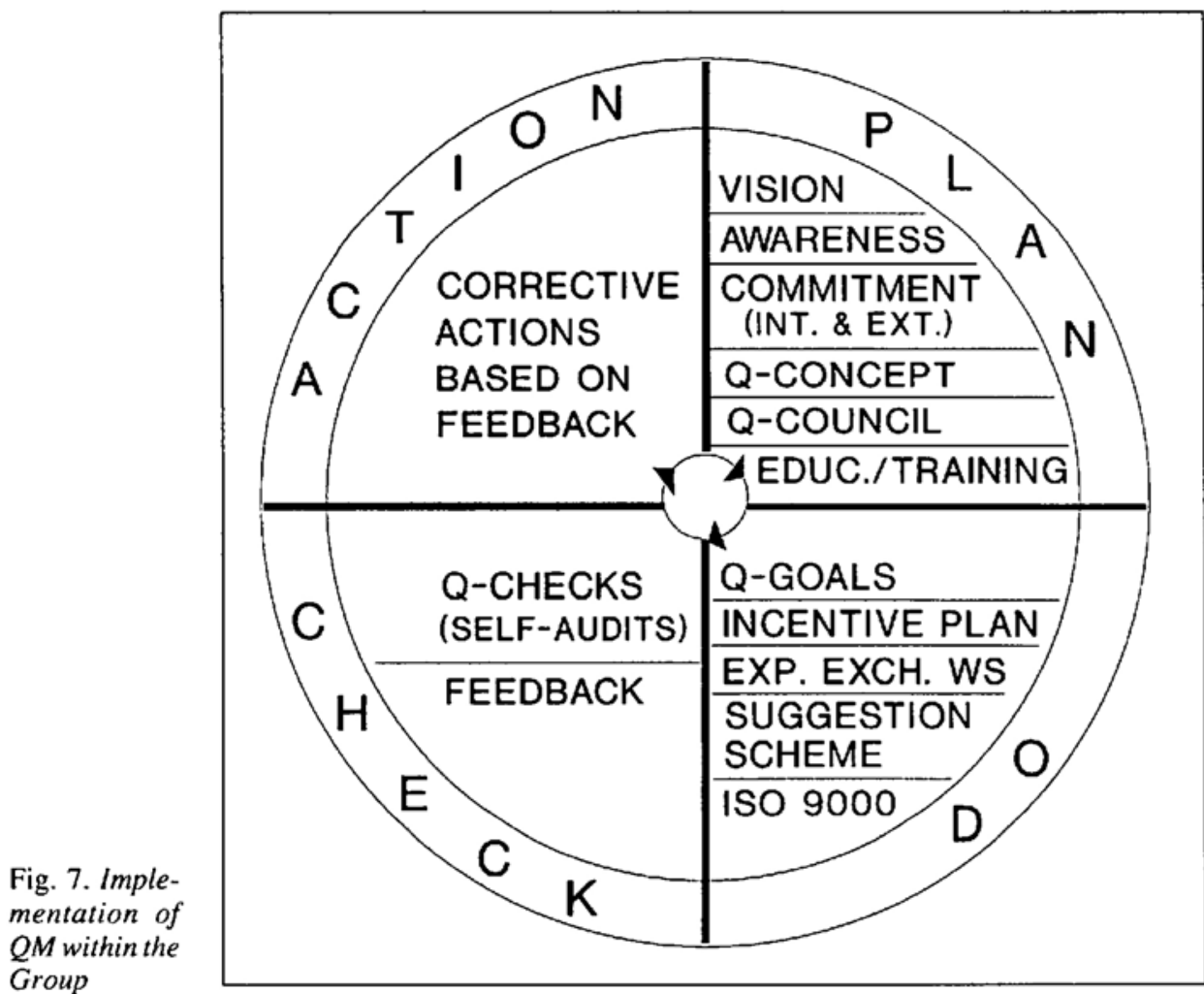

Wir wählten den bereits erwähnten Deming-Kreis als eine geeignete Strategie, welche diese drei Bedingungen erfüllt.

1990 begann die Planungsphase. Qualität wurde als Teil unserer VISION definiert und verankert. In Seminaren für das Top-Management hatten 500 des obersten Kaders des Konzerns die Möglichkeit, während eines halben Tages dieses Thema ziemlich ausführlich zu besprechen, mehr als zweihundert TQM-Instruktoren wurden weltweit ausgebildet. In der Umsetzungsphase letztes Jahr, wurden alle Einheiten weltweit gebeten, Qualitätsziele zu bestimmen sowie die Massstäbe für deren Messung festzulegen. Wir sind dabei, unser Vorschlagswesen komplett zu revidieren. Die Einführung der ISO-Norm wurde und wird gefördert. Dieses Jahr fangen wir nun mit der Check-Phase an: sämtliche Einheiten weltweit sind gebeten worden, 'self-assessments' durchzuführen, wobei sie zwischen dem MalcolmBaldrige-Modell oder dem europäischen TQM-Modell frei auswählen dürfen.

Was haben wir bis jetzt erreicht? Es ist nicht möglich, die zahlreichen Beispiele in ihren Einzelheiten zu besprechen. Lediglich einige wenige davon sind in der Tabelle aufgeführt.

Die Umsetzung von Qualitätsmanagement ist keineswegs einfach. Die inherente Schwierigkeit dieser Aufgabe wird Ihnen aus dem folgenden Zitat sofort ersichtlich:

'It must be remembered that there is nothing more difficult to plan, more doubtful of success, nor more dangerous to manage than the creation of a new system. For the initiator has the enmity of all who would profit by the preservation of the old and merely lukewarm defenders in those who would gain by the new.

Nicolo Machiavelli

Am Anfang diese Referates erwähnte ich den Bestseller 'Auf der Suche nach Spitzenleistungen' von Peters und Waterman. Sie werden sich erinnern, dass in diesem Buch 43 amerikanische Firmen als vorbildlich dargestellt wurden. Weniger bekannt ist die Tatsache, dass eine Mehrheit dieser exzellenten Unternehmen heute bereits verschwunden ist. Die Frage, die ein Unternehmen sich stellen muss, ist also nicht, ob es Qualitätsmanagement einführen will, sondern ob es sich leisten kann, es nicht zu tun, wenn das Unternehmen wettbewerbsfähig und erfolgreich bleiben will - und das ist was wir ja alle wollen. 